\title{
Influence of methylmercaptan on the bonding strength of autopolymerizing reline resins to a heat-polymerized denture base resin
}

\author{
Takuya OHKUBO, Makoto OIZUMI and Takuya KOBAYASHI \\ Department of Removable Prosthodontics, School of Dentistry, Iwate Medical University, 1-19 Uchimaru, Morioka 020-8505, Japan \\ Corresponding author, Takuya OHKUBO; E-mail: takuyaoh@iwate-med.ac.jp
}

\begin{abstract}
Effects of methylmercaptan solution (MS), a volatile sulfur compound produced by gram-negative oral microorganisms, on the adhesion of reline denture resins were investigated. For this purpose, a total of 120 disk-shaped specimens prepared from a heatpolymerized denture base resin (Acron) immersed in MS of different concentrations (0.01, 0.1, and 1.0 mol) as well as in purified water as a control at $37^{\circ} \mathrm{C}$ for 4 weeks. Each of three commercial autopolymerizing reline resins (Rebaron, Mild Rebaron, and Tokuyama Rebase II) was bonded to a specimen. The shear bond strength tests were conducted for the specimens, with and without the application of a primer. The bond strength with $1.0 \mathrm{~mol} \mathrm{MS}$ was significantly lower than those with other solutions $(p<0.05)$. The primer application had a significant positive effect on the bond strength. The debonded Acron surfaces showed evidence of incomplete polymerization. The results suggested a potential adhesion-inhibiting effect of the MS on relined dentures.
\end{abstract}

Keywords: Hard reline resin, Methylmercaptan, Shear bond strength

Received Oct 22, 2008: Accepted Jan 7, 2009

\section{INTRODUCTION}

Elderly patients often require prompt treatment of masticatory disorders. For ill-fitting removable dentures, direct reline has been shown to be effective for improving the stability, support, and retention. Direct reline of denture bases in the mouth itself is frequently undertaken chairside procedure that involves the use of autopolymerized acrylic resins. This method is inexpensive and does not require laboratory procedures.

For each relining procedure, its success hinges chiefly on the bond strength between the reline resin and the denture base resin - which has been plagued with less-than-satisfactory bond strength performance ${ }^{1-3)}$. The weak bond strength causes separation at the interface between these materials, followed by stain penetration at the interface. Against this backdrop, dentures that have been used for a prolonged period are prone to adhesive failure. This is because after prolonged use, dentures might develop microcracks because of repeated and forceful flexion, and these cracks may facilitate microorganism invasion or staining ${ }^{2,4,5)}$.

Some studies examined the bond strength between reline resins and denture base resins, wide-ranging attempts have been made to increase the strength by using mechanical surface treatments such as roughening, and chemical surface treatments with methyl methacrylate (MMA) monomers or certain organic solvents of the denture base resin ${ }^{6-13)}$. Other studies examined the influences of immersing denture base resins in water, olive oil, or milk on the bond strength between denture base resins and reline resins ${ }^{14-18)}$. However, to date, the causes of bond failure have not been sufficiently elucidated.

When the surface of a denture that has been used for a long period is ground with a bur, malodor is frequently detected. Many of the microorganisms in the oral flora produce odors under in vitro conditions. Gram-negative oral microorganisms produce volatile sulfur compounds (VSCs), including hydrogen sulfide and methylmercaptan ${ }^{19-23)}$. VSCs are presumably produced by the oral microorganisms that invade the denture base material, and these compounds probably inhibit adhesion between the reline resins and the denture base resins. In this study, the focus would be on methylmercaptan, which is one of the VSCs produced in the mouth and is used as a chain-transfer agent in industrial polymerization processes ${ }^{24}$. Chaintransfer agents stop the elongation of a molecular chain by transferring an atom to the active radical located at the end of a growing chain during polymerization. These agents are useful for decreasing the molecular weights of the reactants involved in polymerization ${ }^{24}$.

Methylmercaptan is a methanethiol used in the plastics industry as a chain-transfer agent to decrease the molecular weights of the reactants involved in polymerization. It interferes in the radical polymerization of both MMA- and EMA-based reline resins. The AC surface was dissolved by primer treatment, and the shear bond strength of the specimens immersed in the methylmercaptan solutions was lower than that of the specimens immersed in water. The purpose of this study was to evaluate the influence of immersing denture base resin in methylmercaptan solution in order to clarify the effects of methylmercaptan on the shear bond strength between a heat-polymerized denture base resin and three hard autopolymerizing reline resins used in chairside procedures. The hypothesis of the present study was that the immersion of denture base resin in methylmercaptan solution would decrease the shear bond strength between the denture base resin and 
reline resins.

\section{MATERIALS AND METHODS}

Materials used

A heat-polymerized denture base resin (AC) (Acron, GC Corp., Tokyo, Japan), an autopolymerizing MMA-based hard reline material (RE) (Rebaron; GC Corp.), and two autopolymerizing ethyl methacrylate (EMA)-based hard reline materials (MI: Mild Rebaron, GC Corp. and TO: Tokuyama Rebase II, Tokuyama Dental, Tokyo, Japan) were used (Table 1).

Besides, two denture primers - one dichloromethane-based (Denture primer, GC Corp.) and the other ethyl acetate-based (Tokuyama Rebase II adhesive, Tokuyama Dental) - were used as the primers for the denture base resin surface according to the manufacturer's instructions.

\section{Specimen preparation}

A total of 120 disk-shaped specimens (diameter: 10.0 $\mathrm{mm}$; thickness: $5.0 \mathrm{~mm}$ ) were prepared from the denture base resin according to the Boucher's manual ${ }^{25}$ ) and polymerized in a constant-temperature water bath (Curetron-1, Nishikawa, Tokyo, Japan) at $74^{\circ} \mathrm{C}$ for 8 hours ${ }^{25)}$. All the specimens were embedded in an acrylic tube using an autopolymerizing resin (Unifast II, GC Corp.), and their surfaces were polished with \#600 SiC abrasive paper.

For the methylmercaptan solutions, three different concentrations of $0.01, \quad 0.1, \quad$ and $1.0 \mathrm{~mol}$ (methylmercaptan sodium salt (ca. 15\% in water), Tokyo Chemical Industry Corp. Ltd., Tokyo, Japan) were prepared. The specimens were then randomly divided into four immersion groups $(n=30)$, namely the three methylmercaptan solutions of different concentrations and the purified water as a control. All specimens were immersed in their respective solutions at $37^{\circ} \mathrm{C}$ for 4 weeks.
Shear bond strength test

For the shear bond strength test, 30 specimens from each methylmercaptan solution were divided into three groups according to the reline material used $(n=10)$. Half of the specimens were treated with the primer, and the remaining specimens were not. A piece of double-sided tape (MAXON No. 200, Holbein Art Materials, Osaka, Japan) with a $4.0 \mathrm{~mm}$ diameter hole was placed over the disk to define the bonding area (Fig. 1). The specimen surfaces treated with and
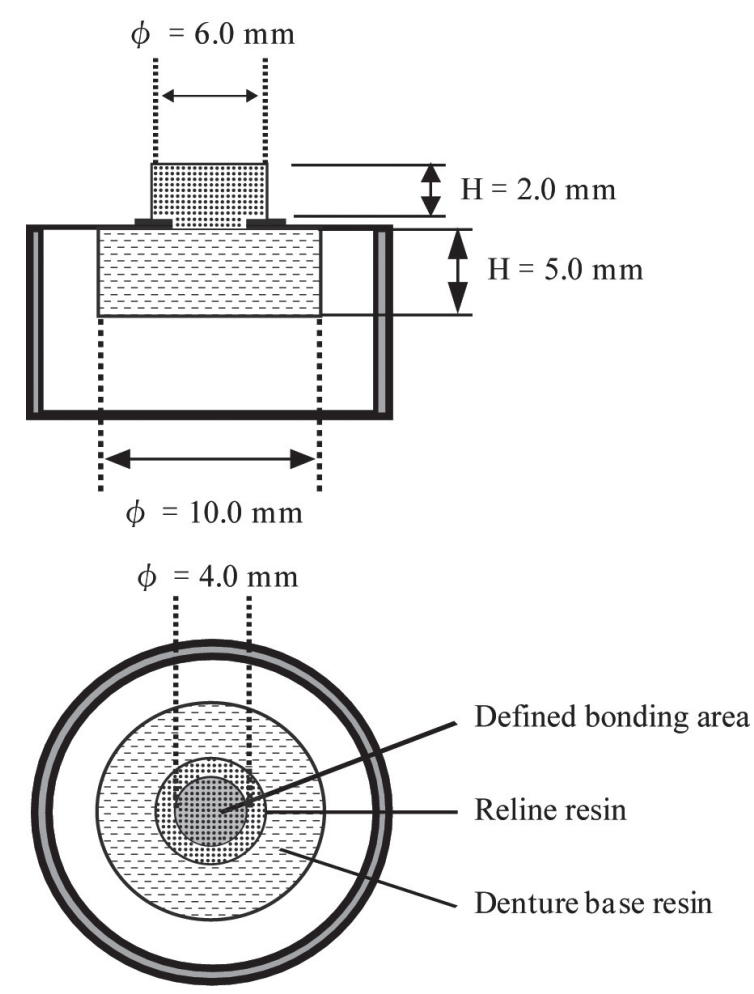

Fig. 1 Schematic illustration of a specimen.

Table 1 Materials used in this study

\begin{tabular}{|c|c|c|c|c|c|c|}
\hline \multirow{2}{*}{ Material } & \multirow{2}{*}{ Code } & \multirow{2}{*}{$\begin{array}{c}\text { Lot No. } \\
\text { (Powder / Liquid) }\end{array}$} & \multirow{2}{*}{ Manufacture } & \multicolumn{3}{|c|}{ Main Component } \\
\hline & & & & Powder & Liquid & Primer \\
\hline
\end{tabular}

Heat-polymerized denture base resin

Acron $\quad$ AC $0512152 / 0510112 \quad$ GC $\quad$ PMMA $\quad$ MMA

Auto-Polymerizing hard-type chairside reline resin

$\begin{array}{lllclll}\text { Rebaron } & \text { RE } & 0705182 / 0705141 & \text { GC } & \text { PMMA } & \text { MMA } & \text { None (Dichloromethane) } \\ \text { Mild Rebaron } & \text { MI } & 0704134 / 0704122 & \text { GC } & \text { PEMA } & \text { EMA } & \text { Dichloromethane } \\ \text { Tokuyama Rebase } & \text { TO } & \text { F15136 } & \text { Tokuyama Dental } & \text { PEMA } & \text { AAEMA } & \text { Ethyl acetate }\end{array}$

PMMA: poly (methyl methacrylate); PEMA: poly (ethyl methacrylate); AAEMA: acetoacetoxyethyl methacrylate 
without the corresponding denture primer were attached to a Teflon tube (diameter, $6.0 \mathrm{~mm}$; height, $2.0 \mathrm{~mm}$ ). The dichloromethane was used as RE primer, because of evaluation for the effect of the primer and according the manufacturer's suggestion.

The three reline materials were mixed according to their manufacturer's instructions. After insertion into the Teflon tubes, the reline materials were polymerized on a bench at room temperature under no pressure. After immersion in water at $37^{\circ} \mathrm{C}$ for 24 hours, the shear bond strengths of all the specimens were determined using a universal testing machine (EZ-S, Shimadzu, Kyoto, Japan) at a crosshead speed of 1.0 $\mathrm{mm} / \mathrm{min}$. Shear bond strength was calculated as the maximum load during the shear test divided by the defined bonding area.

\section{Fracture surface analysis}

After the shear test, all the fractured surfaces were macroscopically analyzed to classify the fracture patterns. Fractures on the AC surfaces were observed through a scanning electron microscope (SEM) (Miniscope TM-1000, Hitachi High-Technologies Corp., Tokyo, Japan).

\section{Statistical analysis}

Statistical analysis was performed using a statistical software (JMP ver. 6.0, SAS, Cary, NC, USA). The shear bond strength of each reline resin was statistically analyzed by two-way analysis of variance (ANOVA) in combination with Tukey's multiple comparison test. Significance level was set at $\alpha=0.05$.

\section{RESULTS}

The means and standard deviations of shear bond strength are summarized in Table 2. The results of two-way ANOVA of each reline resin indicated that both the immersion medium (methylmercaptan solutions of different concentrations) and usage of primer significantly affected the shear bond strength between the denture base resin and the reline resin. No interaction was observed between the immersion medium and the primer. The shear bond strength after immersion in the $1.0 \mathrm{~mol}$ methylmercaptan solution, both with and without primer, was significantly lower than that after immersion in the other solutions (Table 2). The shear bond strength, regardless of the immersion medium, was greater when the primer was used than when it was not, except for the shear bond

Table 2 Means and standard deviations of shear bond strength (MPa) of the test groups in this study $(n=5)$.

\begin{tabular}{|c|c|c|c|c|c|}
\hline \multirow{2}{*}{ Code } & \multirow{2}{*}{ Primer } & \multirow{2}{*}{ Control } & \multicolumn{3}{|c|}{ methylmercaptan solution } \\
\hline & & & $0.01 \mathrm{~mol}$ & $0.1 \mathrm{~mol}$ & $1.0 \mathrm{~mol}$ \\
\hline \multirow[t]{2}{*}{$\mathrm{RE}$} & without & $16.81 \pm 1.42^{\mathrm{a}}$ & $15.63 \pm 2.27^{\mathrm{a}}$ & $15.24 \pm 1.52^{\mathrm{a}}$ & $5.44 \pm 1.26^{b}$ \\
\hline & with & $18.23 \pm 2.16^{\mathrm{a}}$ & $17.49 \pm 1.58^{\mathrm{a}}$ & $16.72 \pm 2.05^{\mathrm{a}}$ & $10.43 \pm 1.46^{\mathrm{c}}$ \\
\hline \multirow[t]{2}{*}{ MI } & without & $7.88 \pm 0.77^{\mathrm{d}}$ & $7.57 \pm 1.21^{\mathrm{d}}$ & $6.68 \pm 1.61^{\mathrm{d}}$ & $2.12 \pm 0.14^{\mathrm{e}}$ \\
\hline & with & $13.50 \pm 1.01^{\mathrm{f}}$ & $13.40 \pm 2.54^{\mathrm{f}}$ & $13.40 \pm 2.42^{\mathrm{f}}$ & $8.85 \pm 1.38^{d}$ \\
\hline \multirow[t]{2}{*}{$\mathrm{TO}$} & without & $5.32 \pm 0.60^{\mathrm{g}, \mathrm{h}}$ & $4.71 \pm 0.62^{\mathrm{g}, \mathrm{h}}$ & $3.64 \pm 0.79^{\mathrm{h}, \mathrm{i}}$ & $2.37 \pm 0.28^{i}$ \\
\hline & with & $5.57 \pm 0.55^{\mathrm{g}, \mathrm{h}}$ & $6.17 \pm 1.31^{\mathrm{g}}$ & $6.03 \pm 2.42^{\mathrm{g}}$ & $2.34 \pm 0.70^{\mathrm{i}}$ \\
\hline
\end{tabular}

Groups identified with the same superscripts are not significantly different $(p<0.05)$.

Table 3 Fracture patterns after the shear bond strength test

\begin{tabular}{|c|c|c|c|c|c|}
\hline \multirow{2}{*}{ Code } & \multirow{2}{*}{ Primer } & \multirow{2}{*}{ Control } & \multicolumn{3}{|c|}{ methylmercaptan solution } \\
\hline & & & $0.01 \mathrm{~mol}$ & $0.1 \mathrm{~mol}$ & $1.0 \mathrm{~mol}$ \\
\hline \multirow[t]{2}{*}{$\mathrm{RE}$} & without & $0 / 2(2) / 3$ & $0 / 3(2) / 2$ & $0 / 5(0) / 0$ & $0 / 0(0) / 5$ \\
\hline & with & $1 / 3(2) / 1$ & $0 / 4(0) / 1$ & $0 / 4(0) / 1$ & $0 / 4(0) / 1$ \\
\hline \multirow[t]{2}{*}{ MI } & without & $0 / 0(0) / 5$ & $0 / 0(0) / 5$ & $0 / 0(0) / 5$ & $0 / 0(0) / 5$ \\
\hline & with & $0 / 1(0) / 4$ & $0 / 1(0) / 4$ & $0 / 1(1) / 4$ & $0 / 0(0) / 5$ \\
\hline \multirow[t]{2}{*}{ TO } & without & $0 / 0(0) / 5$ & $0 / 0(0) / 5$ & $0 / 0(0) / 5$ & $0 / 0(0) / 5$ \\
\hline & with & $0 / 1(0) / 4$ & $0 / 0(0) / 5$ & $0 / 0(0) / 5$ & $0 / 0(0) / 5$ \\
\hline
\end{tabular}

Cohesive fracture of reline resin / mixed fracture (mixed fracture of AC side) / interfacial fracture 

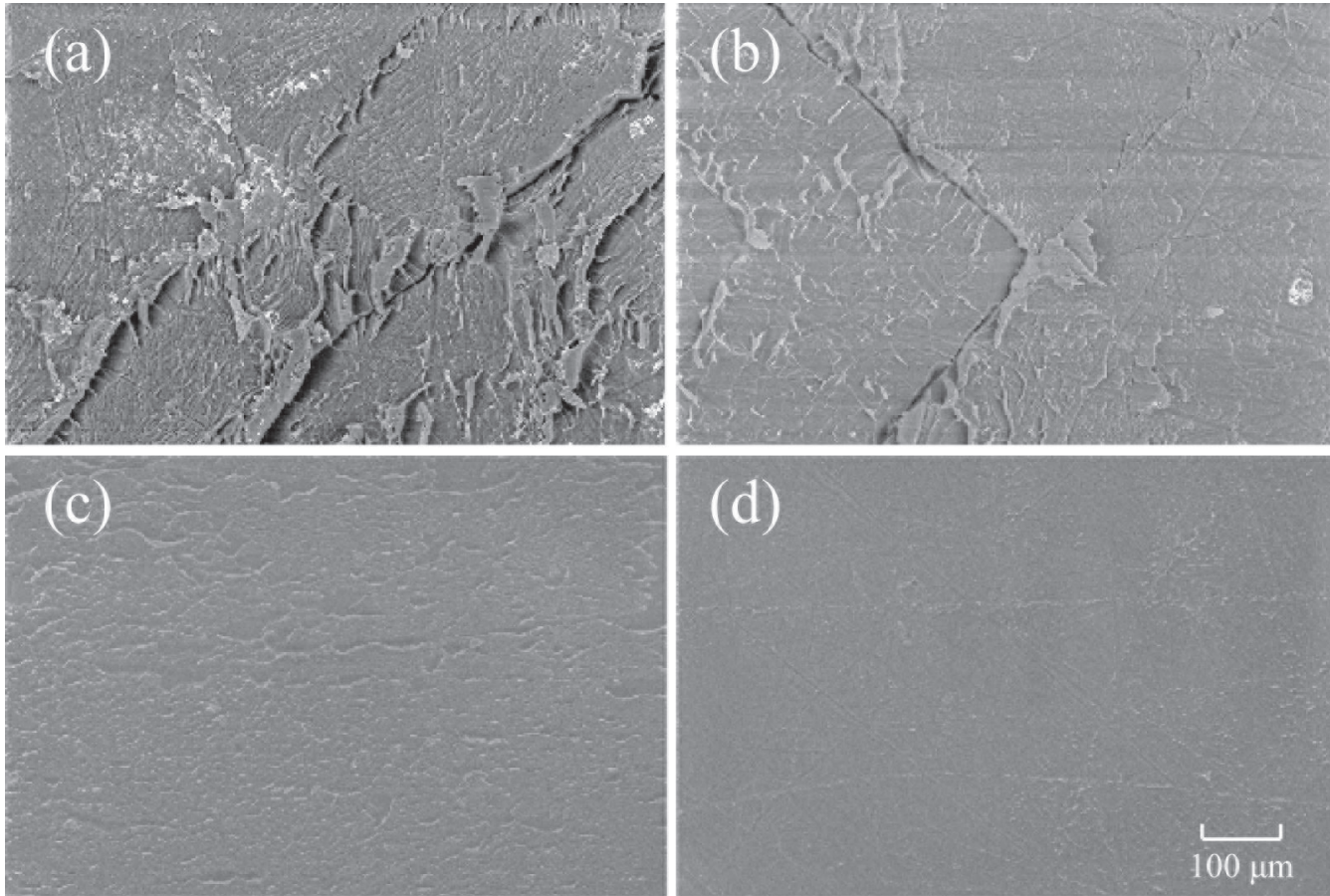

Fig. 2 SEM images of the fracture surfaces on AC of RE specimens immersed in purified water and 1.0 mol methylmercaptan solution: (a) RE with primer immersed in purified water; (b) RE without primer immersed in purified water; (c) RE with primer immersed in 1.0mol methylmercaptan solution; and (d) RE without primer immersed in 1.0-mol methylmercaptan solution.
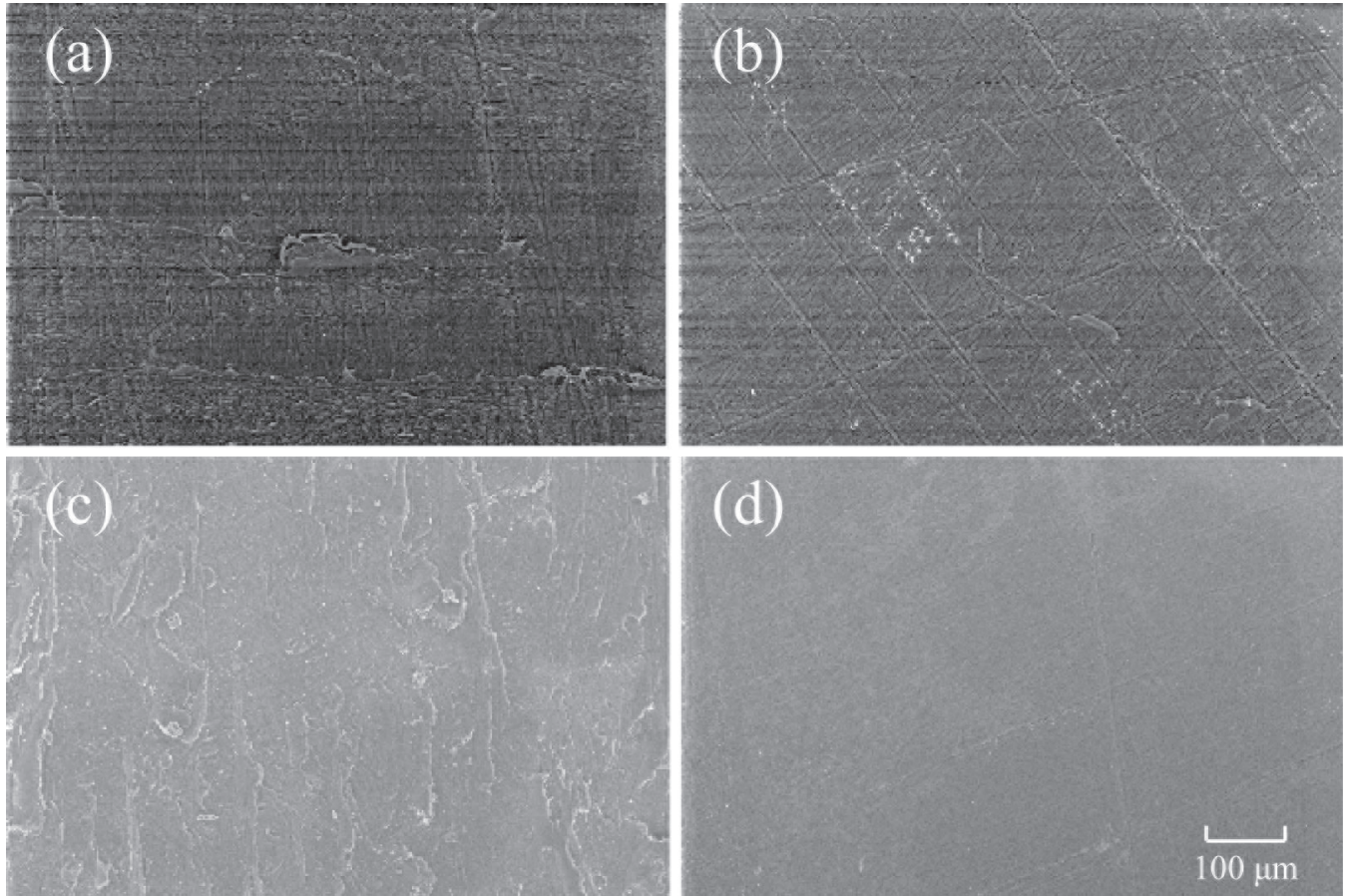

Fig. 3 SEM images of the fracture surfaces on AC of MI specimens immersed in purified water and 1.0 mol methylmercaptan solution: (a) MI with primer immersed in purified water; (b) MI without primer immersed in purified water; (c) MI with primer immersed in 1.0mol methylmercaptan solution; and (d) MI without primer immersed in 1.0-mol methylmercaptan solution. 

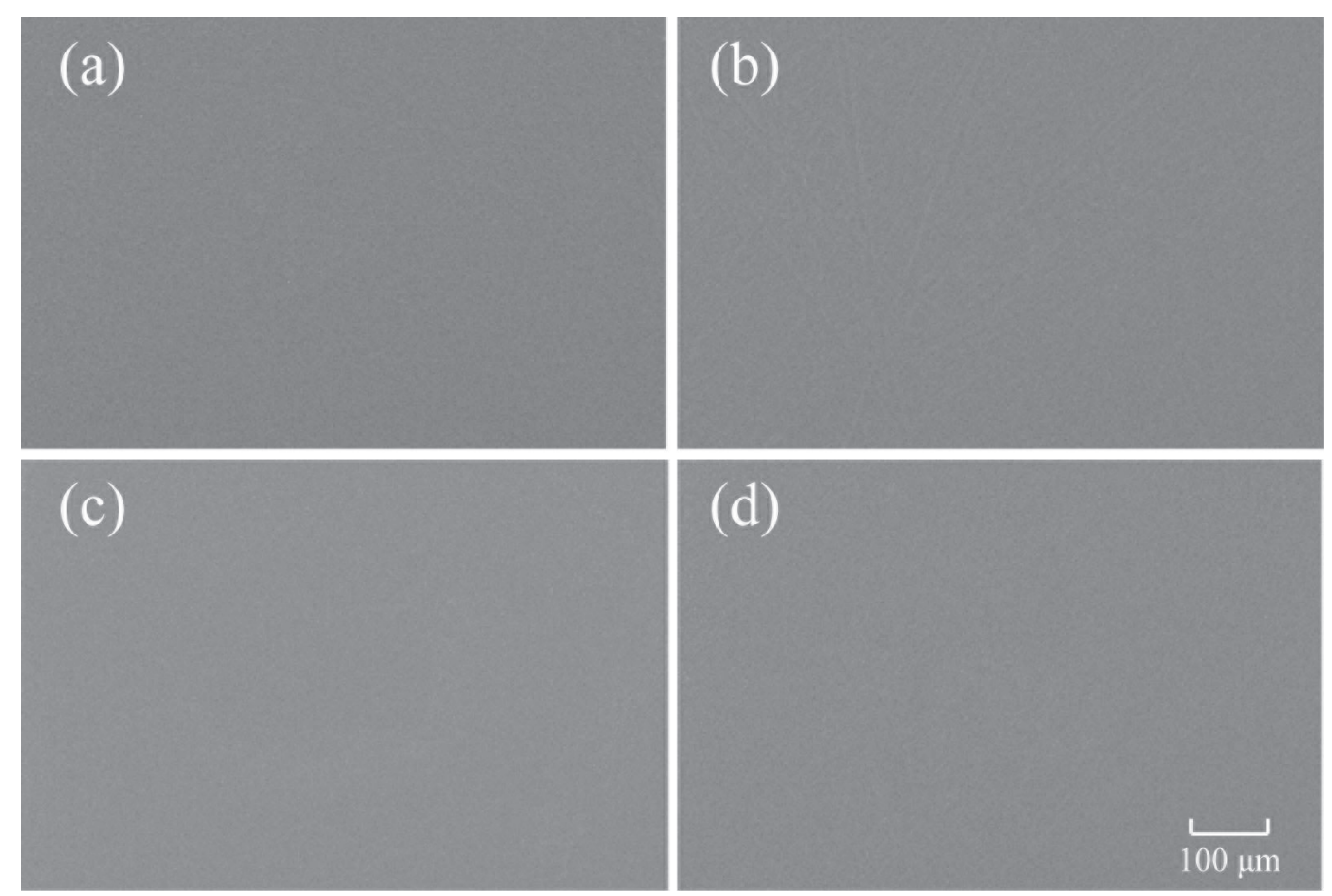

Fig. 4 SEM images of the fracture surfaces on AC of TO specimens immersed in purified water and 1.0 mol methylmercaptan solution: (a) TO with primer immersed in purified water; (b) TO without primer immersed in purified water; (c) TO with primer immersed in 1.0mol methylmercaptan solution; and (d) TO without primer immersed in 1.0-mol methylmercaptan solution.

strength of TO after immersion in the $1.0 \mathrm{~mol}$ solution.

The fracture patterns obtained after the shear bond test are classified in Table 3. Interfacial fractures were most commonly observed. When the primer was not used, all fractures, except for $10 \mathrm{RE}$ specimens, were interfacial fractures. When the primer was applied, mixed fractures were observed in $15 \mathrm{RE}$, three MI, and one TO specimens, while cohesive fracture was noted in one RE specimen.

SEM images of the fracture surfaces on AC immersed in purified water and $1.0 \mathrm{~mol}$ methylmercaptan solution are shown in Figs. 2, 3 and 4. The surfaces of RE and MI specimens in $1.0 \mathrm{~mol}$ methylmercaptan solution to which the primer was not applied developed interfacial fractures. The remaining $\mathrm{RE}$ and MI specimens to which the primer was applied had rough surfaces due to residues of the reline resin. The surfaces of the TO specimens with and without the primer, except for a control specimen with the primer, showed interfacial fractures. No residues of the reline resin were observed.

\section{DISCUSSION}

Results of the shear bond strength test showed that not only MMA-, but also EMA-based, reline resins immersed in the $1.0 \mathrm{~mol}$ methylmercaptan solution exhibited significantly lower bond strengths than the resins immersed in purified water, regardless of primer application. Methylmercaptan is a methanethiol used in the plastics industry as a chain-transfer agent to decrease the molecular weights of the reactants involved in polymerization. It hence interferes in the radical polymerization of both MMA- and EMA-based reline resins. Although the AC surfaces were dissolved by primer treatment, the shear bond strengths of the specimens immersed in three metylmercaptan solutions of varying concentrations were lower than those immersed in water. These results suggest that the methylmercaptan adsorbed on the AC resin surfaces interfered in the polymerization of the reline resins. Indeed, small areas of unpolymerized reline resins were observed on the SEM images of the debonded AC surfaces of the specimens immersed in the methylmercaptan solutions.

On the fracture patterns of specimens immersed in water without primer treatment, two out of five $\mathrm{RE}$ specimens exhibited mixed fractures, while all MI and TO specimens exhibited interfacial fractures (Table 3). These results showed that the $\mathrm{RE}$ resin had a greater monomer-penetrating activity than the MI and TO resins. It is noteworthy that the molecular weight of the MMA monomer of $\mathrm{RE}$ resin was lower than those of the EMA-based monomers of MI and TO resins. This 
difference could have thus accounted for the difference in monomer penetration from the reline resins into the denture base resin ${ }^{24)}$. Therefore, by virtue of its higher monomer-penetrating capability, RE is usually used without primers. Conversely, MI is usually used with a dichloromethane-based primer and TO with an ethyl acetate-based primer, since EMA monomers -unlike MMA monomers- do not penetrate the denture base resin easily. It is also noteworthy that the dichloromethane-based primer was more effective than the ethyl acetate-based primer ${ }^{12)}$. Hence, the difference in bond strength with or without the use of primer was more pronounced in the case of MI than in the case of TO.

The SEM images of RE and MI specimens treated with primer revealed rough surfaces due to residual reline resins. The primer dissolved the AC surface and methylmercaptan penetrated into the AC surface might be eliminated by the evaporation of the primer. Significant differences were observed between the RE specimens with and without primer treatment after they were immersed in the $1.0 \mathrm{~mol}$ methylmercaptan solution. The AC surface immersed in the highconcentration methylmercaptan solution, whereas it was only slightly so for the AC surface immersed in the low-concentration methylmercaptan solution. Consequently, methylmercaptan that had deeply penetrated AC could not be completely removed by the primer and hence interfered in the polymerization of the reline resins. Indeed, fractures observed after immersion in the high concentration methylmercaptan solution were almost exclusively interfacial failure.

Some reports have examined the penetration of bacteria into denture acrylic resins ${ }^{26,27)}$. Many bacterial species penetrate the surface of acrylic resins up to a depth of $3 \mathrm{~mm}$. In particular gram-negative bacteria produce methylmercaptan from l-methionine, an amino $\operatorname{acid}^{20)}$. Dentures that have been used for a prolonged period and porous dentures are more easily penetrated by bacteria, which deeply invade the dentures and produce methylmercaptan. In the present study, the purpose of immersing denture base resin in methylmercaptan solutions of varying concentrations was to simulate the conditions under which methylmercaptan is produced in the dentures.

In conclusion, results of the present study suggested that dentures that have been used for a long time, and are hence potentially deeply invaded by microorganisms, are difficult to reline. Microorganisms produce methylmercaptan, which causes the separation of the reline resin from the denture base resin, even after the dissolution of the denture surface by primer treatment.

\section{ACKNOWLEDGMENTS}

The authors would like to thank Professor Tetsuya Suzuki, Department of Removable Prosthodontics, Iwate Medical University, for his editing this manuscript. The authors would also like to thank
Associate Professor Hidekazu Takahashi, Advanced Biomaterials, Department of Restorative Science, Graduate School, Tokyo Medical and Dental University, for his support and advice.

\section{REFERENCES}

1) Beyli MS, von Fraunhofer JA. Repair of fractured acrylic resin. J Prosthet Dent 1980; 44: 497-503.

2) Berge M. Bending strength of intact and repaired denture base resins. Acta Odontol Scand 1983; 41: 187-191.

3) Bunch J, Johnson GH, Brudvik JS. Evaluation of hard direct reline resins. J Prosthet Dent 1987; 57: 512-519.

4) Arena CA, Evans DB, Hilton TJ. A comparison of bond strengths among chairside hard reline materials. J Prosthet Dent 1993; 70: 126-131.

5) Haywood J, Basker RM, Watson CJ, Wood DJ. A comparison of three hard chairside denture reline materials. Part I. Clinical evaluation. Eur J Prosthodont Restor Dent 2003; 11: 157-163.

6) Matsumura H, Tanoue N, Kawasaki K, Atsuta M. Clinical evaluation of a chemically cured hard denture relining material. J Oral Rehabil 2001; 28: 640-644.

7) Arima T, Nikawa H, Hamada T, Harsini. Composition and effect of denture base resin surface primers for reline acrylic resins. J Prosthet Dent 1996; 75: 457-462.

8) Leles CR, Machado AL, Vergani CE, Giampaolo ET, Pavarina AC. Bonding strength between a hard chairside reline resin and a denture base material as influenced by surface treatment. J Oral Rehabil 2001; 28: 1153-1157.

9) Takahashi Y, Chai J. Assessment of shear bond strength between three denture reline materials and a denture base acrylic resin. Int J Prosthodont 2001; 14: 531-535.

10) Vallittu PK, Lassila VP, Lappalainen R. Wetting the repair surface with methyl methacrylate affects the transverse strength of repaired heat-polymerized resin. J Prosthet Dent 1994; 72: 639-643.

11) Vallittu PK, Ruyter IE. Swelling of poly(methyl methacrylate) resin at the repair joint. Int J Prosthodont 1997; 10: 254-258.

12) Shimizu H, Ikuyama T, Hayakawa E, Tsue F, Takahashi Y. Effects of surface preparation using ethyl acetate on the repair strength of denture base resin. Acta Odontol Scand 2006; 64: 159-163.

13) Seo RS, Murata H, Guang H, Vergani CE, Hamada T. Influence of thermal and mechanical stresses on the strength of intact and relined denture bases. J Prosthet Dent 2006; 96: 59-67.

14) León BL, Del Bel Cury AA, Rodrigues Garcia RC. Water sorption, solubility, and tensile bond strength of resilient denture lining materials polymerized by different methods after thermal cycling. J Prosthet Dent 2005; 93: 282-287.

15) Cucci AL, Vergani CE, Giampaolo ET, Afonso MC. Water sorption, solubility, and bond strength of two autopolymerizing acrylic resins and one heat-polymerizing acrylic resin. J Prosthet Dent 1998; 80: 434-438.

16) Cucci AL, Rached RN, Giampaolo ET, Vergani CE. Tensile bond strengths of hard chairside reline resins as influenced by water storage. J Oral Rehabil 1999; 26: 631-634.

17) Azevedo A, Machado AL, Giampaolo ET, Pavarina AC, Vergani CE. The effect of water immersion on the shear bond strength between chairside reline and denture base acrylic resins. J Prosthodont 2007; 16: 255-262.

18) Sato T, Takahashi H, Hongo T, Hayakawa I. Effect of degradation of denture base resin on bond strength to relining resins. Dent Mater J 2007; 26: 89-95.

19) Persson S, Edlund MB, Claesson R, Carlsson J. The formation of hydrogen sulfide and methyl mercaptan by oral 
bacteria. Oral Microbiol Immunol 1990; 5: 195-201.

20) Fukamachi H, Nakano Y, Okano S, Shibata Y, Abiko Y, Yamashita Y. High production of methyl mercaptan by Lmethionine-alpha-deamino-Y-mercaptomethane lyase from Treponema denticola. Biochem Biophys Res Commun 2005; 331: 127-131.

21) Krespi YP, Shrime MG, Kacker A. The relationship between oral malodor and volatile sulfur compoundproducing bacteria. Otolaryngol Head Neck Surg 2006; 135: 671-676.

22) Tonzetich J, McBride BC. Characterization of volatile sulphur production by pathogenic and non-pathogenic strains of oral bacteroides. Arch Oral Biol 1981; 26: 963969.

23) Goldberg S, Cardash H, Browning H III, Sahly H, Rosenberg M. Isolation of Enterobacteriaceae from the mouth and potential association with malodor. J Dent Res 1997; 76: 1770-1775.

24) Matyjaszewski K, Davis TP. Handbook of radical polymerization, 1st ed, John Wiley \& Sons Inc., New York, 2002, pp.523-628.

25) Zarb GA, Bolender CL, Eckert SE, Jacob RF, Fenton AH, Mericske-Stern R. Boucher's prosthodontic treatment for edentulous patients: complete dentures and implantsupported prostheses, $12^{\text {th }}$ ed, Mosby, St. Louis, 2004, pp.382-426.

26) Chau VB, Saunders TR, Pimsler M, Elfring DR. In-depth disinfection of acrylic resins. J Prosthet Dent 1995; 74: 309313.

27) Walter B, Frank RM. Ultrastructural relationship of denture surfaces, plaque and oral mucosa in denture stomatitis. J Biol Buccale 1985; 13: 145-166. 